\title{
Author Index Vol. 29, No. 5, 1997
}

Aiello, L.P. 354 Ali, R.R. 261

Bhattacharya, S.S. 261 Biedermann, B. 326 Bringmann, A. 326

Miliczek, K.-D. 269 Mohand-Said, S. 290 Mosinger Ogilvie, J. 290 Murata,T. 242

Nisch,W. 269

Coffey,P.J. 305

Deudon-Combe, A. 290 Dreyfus, H. 290

Eckmiller, R. 281 Enzmann, V. 298, 326

Faude, F. 298, 326 Francke, M. 326 Frank, R.N. 341 Funk,R.H.W. 320

Gabel,V.P. 269 Graf,H.G. 269 Guenther,E. 269

Haemmerle, H. 269 Heinemann, U. 326 Hicks, D. 290 Hinton,D.R. 242 Hoefflinger, B. 269

Hunt,D.M. 261

Kimura, H. 242 Kohen,L. 241,298 Kohler, K. 269 Kuhrt,H. 326

Lawrence, J.M. 305 Lund,R.D. 305

Osusky, R. 242

Pannicke, T. 326

Reichel, M.B. 261 Reichelt, W. 326 Reichenbach, A. 326 Ropers, H.H. 252 Ryan, SJ. 242

Sahel,J. 290 Sakamoto, T. 242 Sauvé,Y. 305 Schubert, M. 269 Silverman, M.S. 290 Simonutti, M. 290 Skatchkov, S.N. 326 Spee, C. 242 Stett,A. 269 Stolzenburg, J.-U. 326 Stout, T.J. 242

Tenkova,T. 290 Tran-Minh, D. 290

Weiss, S. 269

Wiedemann, P. 241,298,326

Zrenner, E. 269

KAKGEIl

E-Mail karger@karger.ch

Fax+ 41613061234 http://www.kargcr.ch

(C) 1997 S. Karger AG, Basel

363 